\section{The American Journal of Psychiatry}

May 2020, Volume 177

\section{EDITOR'S NOTE}

365 The Critical Relationship Between Anxiety and Depression Ned H. Kalin

\section{EDITORIALS}

368 Some Comments on Psychedelic Research Alan F. Schatzberg

370 What Does It Mean to Be Transdiagnostic and How Would We Know? Deanna M. Barch

373 Mapping Symptom Clusters to Circuits: Toward Personalizing TMS Targets to Improve Treatment Outcomes in Depression Sean M. Nestor, Daniel M. Blumberger

376 Is Depression Nature or Nurture? Yes Myrna M. Weissman

378 Developmental Differences in Neural Responding to Threat and Safety: Implications for Treating Youths With Anxiety Dylan G. Gee, Sahana Kribakaran

\section{REVIEWS AND OVERVIEWS}

381 Toward Circuit Mechanisms of Pathophysiology in Depression Timothy Spellman, Conor Liston

391 Psychedelics and Psychedelic-Assisted Psychotherapy Collin M. Reiff et al

\section{NEW RESEARCH - ARTICLES}

411 Identification of Common Neural Circuit Disruptions in Emotional Processing Across Psychiatric Disorders Lisa M. McTeague et al.

422 Multimodal Abnormalities of Brain Structure and Function in Majo Depressive Disorder: A Meta-Analysis of Neuroimaging Studies Jodie P. Gray et al.

435 Distinct Symptom-Specific Treatment Targets for Circuit-Based Neuromodulation

Shan $\mathrm{H}$. Siddiqi et al.

447 The Rearing Environment and Risk for Major Depression: A Swedish National High-Risk Home-Reared and Adopted-Away Co-Sibling Control Study

Kenneth S. Kendler et al.

454 Age Differences in the Neural Correlates of Anxiety Disorders: An fMR Study of Response to Learned Threat Andrea L. Gold et al.

\section{COMMUNICATIONS AND UPDATES - LETTERS TO THE EDITOR}

447 Examining the Reach of Smartphone Apps for Depression and Anxiety Akash R. Wasil et al.

\title{
BJPsych

\section{Contents}

CPD Online New from CPD Online

Cochrane Corner Family therapy approaches for anorexia nervosa: a Cochrane Review

Caroline A. Fisher, Sonja Skocic, Kathleen A. Rutherford \&

Sarah E. Hetrick

Round the Corner Is it time to review NICE guidelines on family therapy for anorexia in young people?

Esther WOO

Articles The clinical assessment of acts of violence: mental mechanisms and subjectivity

Rajan Nathan \& Peter Wilson

Articles Complex post-traumatic stress disorder: a new

diagnosis in ICD-11

Chris R. Brewin
Commentary PTSD diagnoses and treatments: closing the gap between ICD-11 and DSM-5

Amy Lehrner \& Rachel Yehuda

Refreshment Brain and pain: old assumptions and new science about chronic pain

George Ikkos \& Parashar Pravin Ramanuj

Articles Borderline personality disorder: part 1 - assessment and diagnosis

Jacqueline Garland \& Stephen Miller

Articles Sexuality and sexual dysfunctions in older people: a forgotten problem

Philip Slack \& Victor M. Aziz

Articles Practicalities of care closer to home: seven key questions for community psychiatrists Prasanna N. de Silva 